\title{
छூ \\ Generation of stable subfemtosecond hard x-ray pulses with optimized nonlinear bunch compression
}

\author{
Senlin Huang, ${ }^{1}$ Yuantao Ding, ${ }^{2, *}$ Zhirong Huang, ${ }^{2}$ and Ji Qiang ${ }^{3}$ \\ ${ }^{1}$ Institute of Heavy Ion Physics, School of Physics, Peking University, Beijing 100871, China \\ ${ }^{2}$ SLAC National Accelerator Laboratory, Menlo Park, California 94025, USA \\ ${ }^{3}$ Lawrence Berkeley National Laboratory, Berkeley, California 94720, USA
}

(Received 7 October 2014; published 15 December 2014)

\begin{abstract}
In this paper, we propose a simple scheme that leverages existing x-ray free-electron laser hardware to produce stable single-spike, subfemtosecond x-ray pulses. By optimizing a high-harmonic radio-frequency linearizer to achieve nonlinear compression of a low-charge $(20 \mathrm{pC})$ electron beam, we obtain a sharp current profile possessing a few-femtosecond full width at half maximum temporal duration. A reverse undulator taper is applied to enable lasing only within the current spike, where longitudinal space charge forces induce an electron beam time-energy chirp. Simulations based on the Linac Coherent Light Source parameters show that stable single-spike x-ray pulses with a duration less than 200 attoseconds can be obtained.
\end{abstract}

DOI: 10.1103/PhysRevSTAB.17.120703

PACS numbers: 41.60.Cr

\section{INTRODUCTION}

At the level of electrons, all atomic, chemical, biological, and material processes involve angstrom-scale systems moving on attosecond (as) time scales. The advent of $\mathrm{x}$-ray free-electron lasers (FELs) [1,2] has opened up the possibility of generating attosecond $\mathrm{x}$-ray pulses with sufficient intensity for studying these ultrafast processes. Many different schemes to produce such pulses have recently been proposed. Since the electron bunch length is usually tens of femtoseconds (fs) long, a common way to produce attosecond $\mathrm{x}$-ray pulses is based on time slicing where only a small fraction of the bunch is selected to lase. This process is typically controlled by either an extremely short optical laser pulse [3-12] or a slotted foil [13]. After this manipulation, the resultant $\mathrm{x}$-ray pulse is much shorter than the whole electron bunch. Another possible beam manipulation technique involves reducing the electron bunch charge, e.g., down to $1 \mathrm{pC}$ [14-16], and significantly compressing the bunch length such that ultrashort $\mathrm{x}$-ray pulses are amplified. Although straightforward in concept, the electron bunch diagnostics at such low charges are challenging, and the required accelerator stability is very tight. Considering these practical limitations, a low-charge operation mode with $20 \mathrm{pC}$ at the Linac Coherent Light Source (LCLS) has been developed [17] with x-ray pulse duration typical of a few femtoseconds [18-20], and similar low-charge modes have also been considered at other

\footnotetext{
*ding@slac.stanford.edu
}

Published by the American Physical Society under the terms of the Creative Commons Attribution 3.0 License. Further distribution of this work must maintain attribution to the author $(s)$ and the published article's title, journal citation, and DOI. facilities under construction including the SwissFEL and European XFEL $[21,22]$. Other proposals based on various harmonic generation configurations [23-26] and modelocking techniques [27-29] have also been studied.

In one of the laser slicing schemes, Saldin, Schneidmiller, and Yurkov [7] proposed the idea of using a reverse (negative) undulator taper to compensate the electron time-energy chirp which is induced by an external few-cycle conventional laser. This scheme was further studied in the XUV and soft-X-ray regime [30] and has been demonstrated at visible wavelengths [31,32]. The chirp-taper idea is very attractive, but adding a fewfemtosecond, high-power optical laser system and a wiggler to an existing x-ray FEL facility is not simple.

In an x-ray FEL facility, the electron bunch is compressed in time to achieve a high peak current. Before the compressor, a high-harmonic radio-frequency (rf) structure is typically adopted to linearize the longitudinal phase space and so that the compressed bunch has a relatively uniform current profile [33,34]. Without this high-harmonic rf linearizer, the compressed bunch could have a "banana" shape in the longitudinal phase space. This was the case for free-electron laser in Hamburg operation at an early stage, as described in Ref. [35], where the current profile has a short high-current leading peak and a long low-current tail. As a result, the FEL pulses were produced mainly from the leading peak and had temporal durations much shorter than the electron bunch. With these results in mind, in this paper we propose operating the high-harmonic rf linearizer in a controlled nonlinear compression mode, rather than as a linearizer, by optimizing its rf amplitude and phase. In this configuration, the rf amplitude of the harmonic structure is lower than the nominal setup for a linearizer, and the resultant phase space has a similar 
banana shape. The peak current, however, can be much higher than the linearizer-off case over a large tuning or jittering range of the main linac rf phase. With such a single-horn high peak current bunch, the downstream longitudinal space charge (LSC) force pushes the electrons near the horn head (tail) to higher (lower) energy and a time-energy chirp will be formed both before and inside the undulator. A reverse undulator taper could then be applied to compensate for the energy chirp within the current spike, which can enable the generation of a stable ultrashort FEL pulse on the subfemtosecond time scale.

In the following sections, we take the LCLS as a representative example to discuss this nonlinear compression mode. In this scheme, only a few rf parameters will be changed compared with the standard LCLS configuration [1]. In Sec. II, we discuss the optimization of the nonlinear bunch compression. In Sec. III, FEL simulations are performed to demonstrate the generation of subfemtosecond x-ray pulses. Particular attention is paid to the impact of rf phase jittering on the FEL performance. The discussion and conclusion are summarized in Sec. IV.

\section{OPTIMIZED NONLINEAR COMPRESSION OF ELECTRON BEAM}

Figure 1 shows a layout of the LCLS, which includes the injector, 1-km-long linac sections with two bunch compressors, the undulator, and transport beam lines. The electron beam is injected into the main linac through DL1 at an energy of $135 \mathrm{MeV}$. It is then compressed twice by the two compressor chicanes $(\mathrm{BC} 1$ and $\mathrm{BC} 2)$ at $220 \mathrm{MeV}$ and $5 \mathrm{GeV}$, respectively. After $\mathrm{BC} 2$, the electrons can either be accelerated to a higher energy up to $15 \mathrm{GeV}$ for hard x-ray FEL operations or decelerated to a lower energy down to about $2.5 \mathrm{GeV}$ for soft $\mathrm{x}$-ray $\mathrm{FEL}$ operations. All the linac sections are $S$-band rf traveling wave structures at $2.856 \mathrm{GHz}$ except one $X$-band rf section (L1X) before $\mathrm{BC} 1$. This $\mathrm{L} 1 \mathrm{X}$, working at $11.424 \mathrm{GHz}$, is a 4th harmonic rf structure with respect to the main accelerating linac rf. It works as the longitudinal phase space linearizer which removes the nonlinear longitudinal phase space curvature and linearizes the bunch compression process in the downstream chicanes [33].

At the LCLS, two short-pulse modes of operation-a low-charge $20 \mathrm{pC}$ mode [17] and an emittance-spoiling foil scheme [13] — have been developed for user experiments with measured $\mathrm{x}$-ray pulse durations of a few femtoseconds [18-20]. Even shorter x-ray pulses, down to $1 \mathrm{fs}$ (FWHM) or shorter, in principle can be obtained at full compression with a $20 \mathrm{pC}$ bunch charge [36] or with even lower charge of $1-3 \mathrm{pC}$ [16]. However, the peak current of the electron beam at full compression is very sensitive to L2 rf phase jittering, making it hard to provide a stable FEL operation for users.

In this work, we prefer a low bunch charge to produce short FEL pulses. We choose the bunch charge to be $20 \mathrm{pC}$, which is a reasonable minimum within the present LCLS diagnostic range. The 4th harmonic $X$-band rf linearizer L1X is optimized for a nonlinear compression mode of operation in the bunch compressors. In this way, modifications to the LCLS nominal parameters are minimized. The configurations of magnets, especially $\mathrm{BC} 1, \mathrm{BC} 2$, and DL2, and the linac L3 are unchanged, which is desirable for fast switching between the normal operation mode and this single-horn mode. Other than the phase and amplitude adjustments to L1X, only the rf phase and amplitude of linac L1S and L2 are adjusted to get the required electron beam energy and chirp for nonlinear compression.

When acting as a linearizer during normal operation, the $\mathrm{L} 1 \mathrm{X}$ rf phase is set near the decelerating crest, about $-160^{\circ}$, with the amplitude of $20 \mathrm{MV}$ [37]. With this setup, a fully compressed electron beam (after BC2) could have a very short bunch length. As mentioned above, however, the current is not stable, since the bunch fluctuates between under- and overcompression even with a small L2 $\mathrm{rf}$ phase jitter. To avoid this jittering issue, we intentionally lower the L1X rf amplitude and adjust the rf phase so that the electron bunch has some residual nonlinear curvature in the middle part of the longitudinal phase space like a banana shape. Then the L2 $\mathrm{rf}$ phase is set to have the bunch fully compressed in the middle part while the bunch head is overcompressed and the bunch tail is still undercompressed. The resultant current profile has a spike from the full-compression region. In this arrangement, the L2 rf phase jitter only leads to a rotation of the electron distribution in the longitudinal phase space. As a result, a stable current spike is always produced as the L2 $\mathrm{rf}$ phase jitters, because different parts of the electron beam near the core part will always get fully compressed.

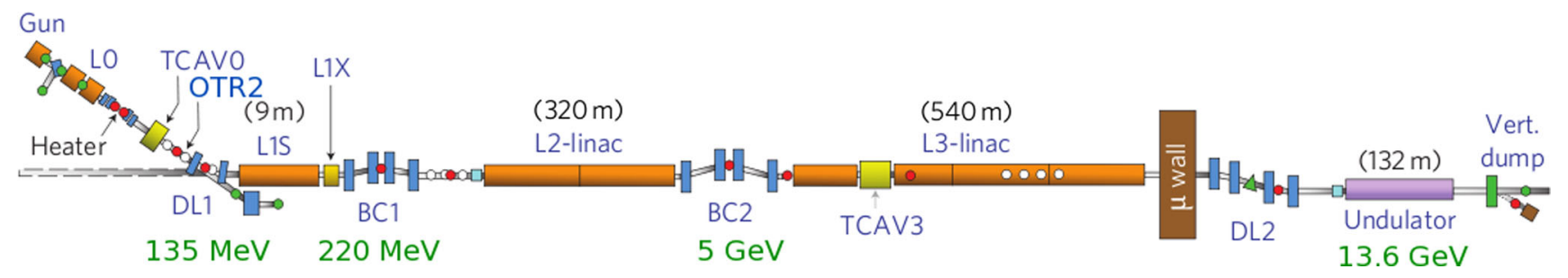

FIG. 1. Layout of the LCLS, with injector sections, main linac $S$-band sections (L1S, L2, L3), one $X$-band linac section (L1X), two bunch compressor chicanes (BC1 and BC2), two dog-leg beam lines (DL1 and DL2), and a 132-m-long undulator. 
TABLE I. The LCLS parameters used in the simulations.

\begin{tabular}{lcc}
\hline \hline Parameter & Value & Unit \\
\hline Bunch charge & 20 & $\mathrm{pC}$ \\
Injector bunch length & 270 & $\mu \mathrm{m}$ \\
Laser heater & 1 & $\mathrm{keV}$ \\
DL1 energy & 135 & $\mathrm{MeV}$ \\
DL1 R56 & 6.3 & $\mathrm{~mm}$ \\
L1S rf phase & -27 & degrees \\
L1X rf phase & -180 & degrees \\
L1X rf amplitude & 15 & $\mathrm{MV}$ \\
BC1 energy & 220 & $\mathrm{MeV}$ \\
BC1 R56 & -45.5 & $\mathrm{~mm}$ \\
L2 rf phase & -35.4 to -34.8 & degrees \\
BC2 energy & 5 & $\mathrm{GeV}$ \\
BC2 R56 & -24.7 & $\mathrm{~mm}$ \\
L3 rf phase & 0 & degrees \\
DL2 energy & 13.6 & $\mathrm{GeV}$ \\
DL2 R56 & 0.133 & $\mathrm{~mm}$ \\
\hline \hline
\end{tabular}

Compared to the L1X off case, where a banana shape of the longitudinal phase space can also be generated, here the proposed scheme with a lower L1X amplitude could have more electrons in the horn region; therefore, a higher peak current can be obtained. Higher current is important not only for achieving a higher FEL efficiency, but also for generating an energy chirp within the current spike through longitudinal space charge forces (discussed below). We first use a fast longitudinal tracking code LITRACK [38] to demonstrate this concept. The main LCLS parameters used in this study are summarized in Table I. The L1S rf phase is decreased from $-22^{\circ}$ in the normal operational mode to $-27^{\circ}$ for higher peak current. The L1X is optimized at an rf phase of $-180^{\circ}$ (decelerating crest phase) and amplitude of $15 \mathrm{MV}$. The optimization ensures that we have a nonlinear phase space after compression to allow for stable operation and to also divert as many electrons as possible into the current spike. Further decreasing the L1S rf phase or increasing the L1X amplitude will lead to even higher current; however, the stability will get worse as the L2 rf phase jitters. Figure 2 shows the final longitudinal phase space and current profile at different L2 rf phases from LITRACK simulations. The phase space has a banana shape, and the full-compression part in the middle of the bunch is shifted a bit when the L2 $\mathrm{rf}$ phase varies from $-34.6^{\circ}$ to $-35.2^{\circ}$. The single current spike, however, remains relatively constant. The typically measured LCLS rf phase jitter is about $0.1^{\circ} \mathrm{rms}$ [39], which ensures this nonlinear compression mode could provide a stable single current spike during operation.

In the LiTRACK simulations, the compression concept is demonstrated, but collective effects such as coherent synchrotron radiation (CSR) and LSC have been ignored. CSR and LSC could affect the beam phase space distribution and should be taken into account, especially after $\mathrm{BC} 2$ where the electron beam has a narrow temporal spike with a high peak current. Therefore, we performed startto-end macroparticle tracking from the cathode to the entrance of the LCLS undulator. The injector, where the electron beam energy is low, is simulated with a full threedimensional space charge code IMPACT-T [40]. At OTR2 (see Fig. 1), where the beam energy is $135 \mathrm{MeV}$, the particle distribution is dumped from IMPACT-T and converted to an input for ELEGANT [41] simulations throughout the linac until the entrance of the undulator. The ELEGANT simulations include the effects of incoherent and coherent synchrotron radiation in bends and LSC in linacs and drifts. The laser heater [42] increases the electron energy spread to about $1 \mathrm{keV}$ in our simulations. This level of energy spread increase, however, has negligible effects on the final phase space, but it is helpful for numerical noise suppression in the simulations. The laser heater could be used to generate a wider current spike by increasing the energy spread, but in this paper we try to set it at minimum to achieve short FEL pulses.

With this setup, we first check the collective effects along the linac. We choose the case with a L2 rf phase of $-34.9^{\circ}$

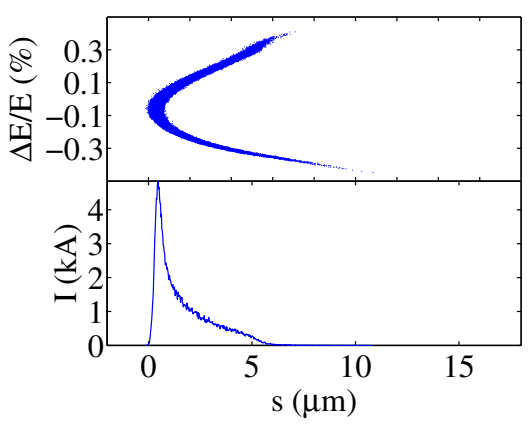

(a) L2@-34.6

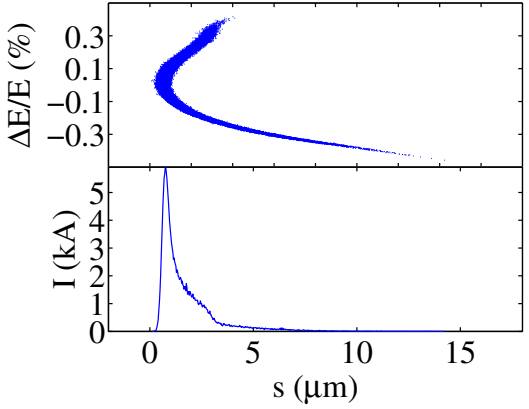

(b) L2@-34.9

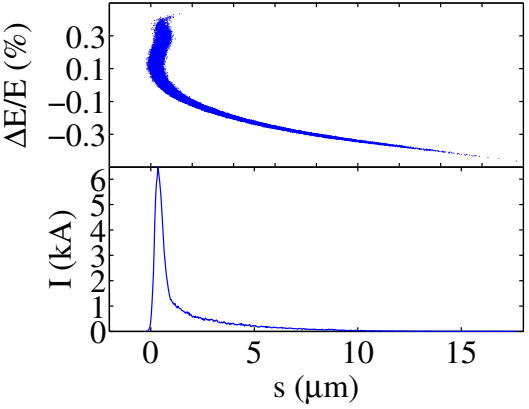

(c) L2@-35.2

FIG. 2. LiTRACK simulations for electron bunch distribution in the longitudinal phase space (upper) and its current profile (lower) at the LCLS undulator entrance. The L2 $\mathrm{rf}$ phase is adjusted from $-34.6^{\circ}$ (a) to $-35.2^{\circ}$ (c), which rotates the longitudinal phase space while maintaining a relatively constant current spike. The L1S rf phase is $-27^{\circ}$, and the L1X phase and amplitude are $-180^{\circ}$ and $15 \mathrm{MV}$, respectively. For the phase space and current plots in this figure and throughout the paper, the bunch head is to the left. 

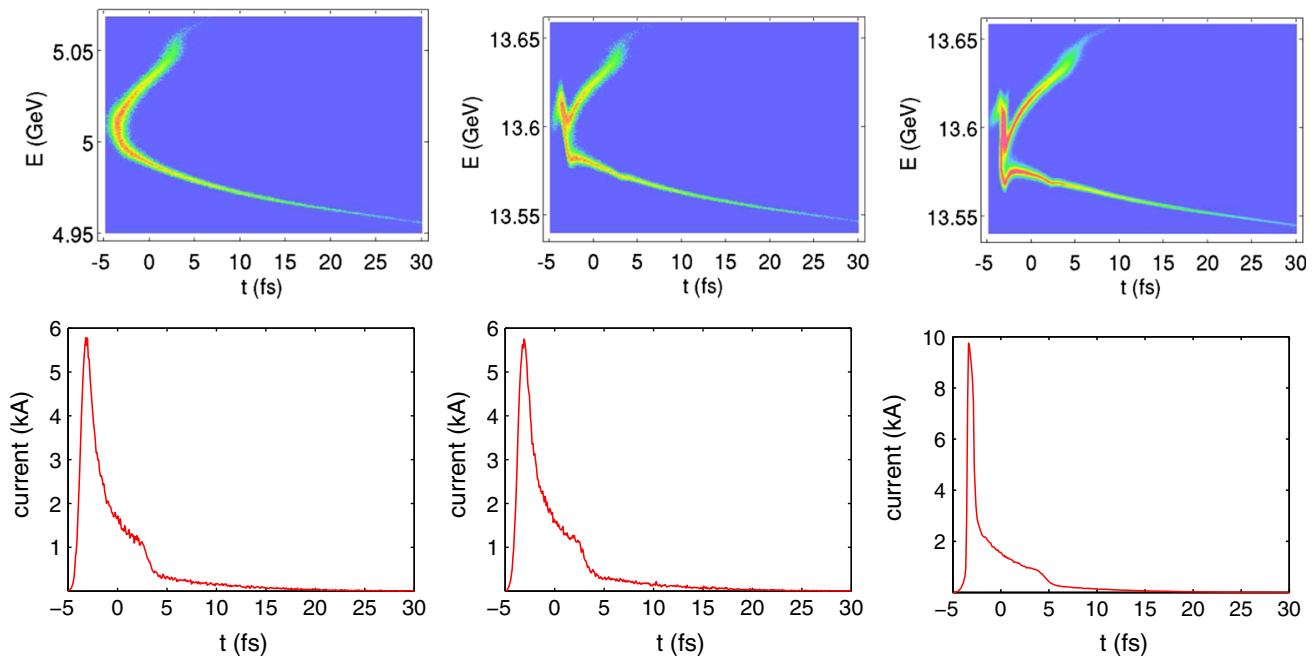

(a) BC2END

(b) L3END

(c) UNDBEG

FIG. 3. ELEGANT tracking results of electron bunch distribution in the longitudinal phase space (upper) and its current profile (lower) at the BC2 end (a), the L3 end (b), and the LCLS undulator entrance (c). The L1S rf phase is $-27^{\circ}$. L1X is with the rf phase of $-180^{\circ}$ and amplitude of $15 \mathrm{MV}$. The L2 $\mathrm{rf}$ phase is $-34.9^{\circ}$.

[to compare with the LITRACK simulation result in Fig. 2(b)] to illustrate the phase space evolution, which can be found in Fig. 3. One can see from the figure that, right after $\mathrm{BC} 2$, the phase space has a banana shape very similar to the LiTRACK results. Then after L3, the fullcompression region in the middle part of the bunch gets an energy chirp, which is mainly due to LSC in the high peak current horn. This interaction continues in the downstream transport beam line, and the positive R56 $(0.133 \mathrm{~mm})$ in DL2 further rotates the phase space, leading to a significant increase of electron peak current [see Fig. 3(c)]. Finally, the horn compresses further $(\sim 1 \mathrm{fs})$ to a peak current
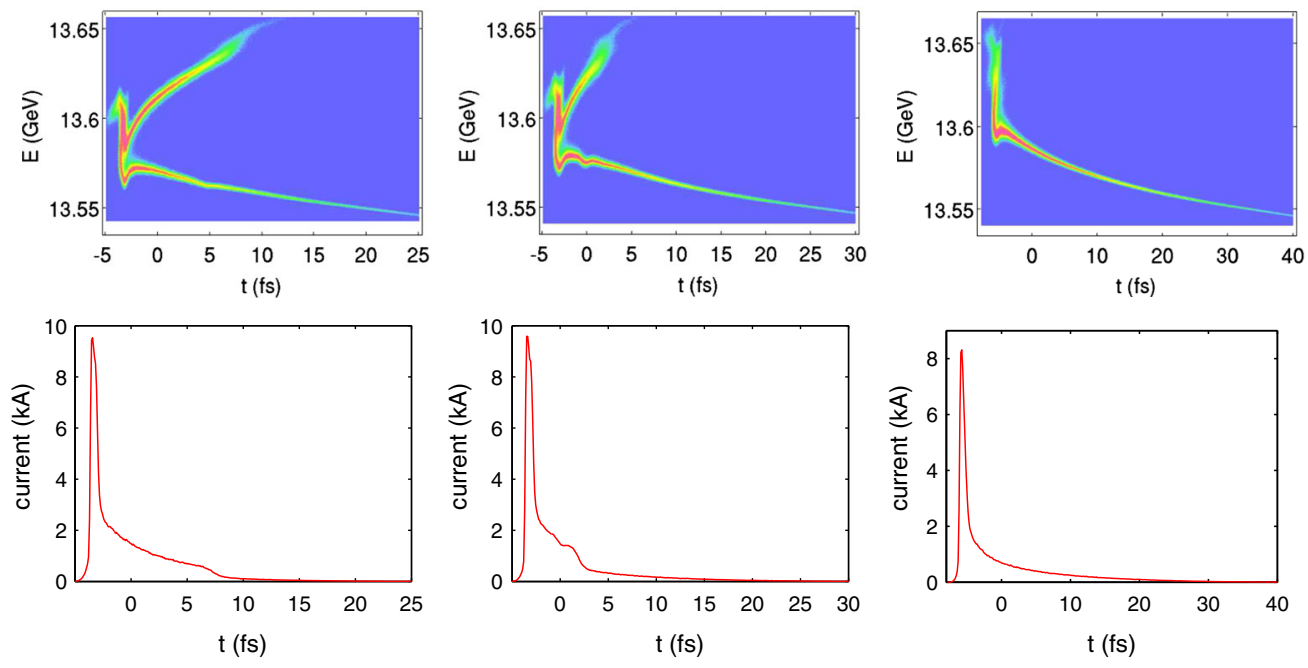

(a) $-34.8^{\circ}$

(b) $-35.0^{\circ}$

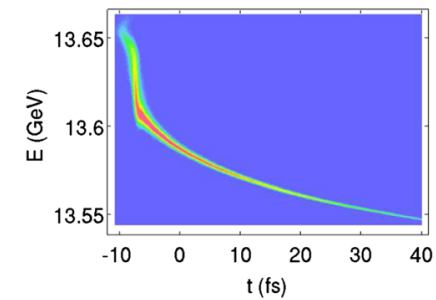

over $9 \mathrm{kA}$. This lays a foundation for generating subfemtosecond FEL pulses.

More simulations have been performed by scanning the L2 rf phase. Some snapshots for the longitudinal phase space and current profile of the electron beam are shown in Fig. 4. In all cases, the electron beam gets compressed further due to the energy chirp induced by the LSC after L3. One can see from the figure that the electron current profiles for all these cases have a single spike with a width close to $1 \mathrm{fs}$ FWHM. When the L2 $\mathrm{rf}$ phase varies from $-34.8^{\circ}$ to $-35.3^{\circ}$, the peak current of the electron beam remains above $8 \mathrm{kA}$. As the L2 $\mathrm{rf}$ phase is further

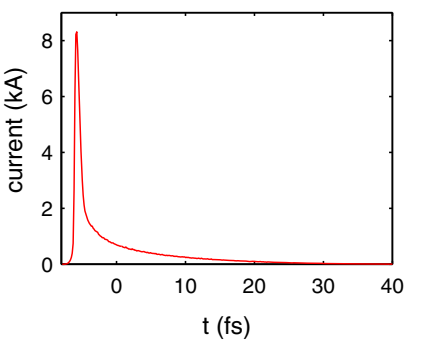

(c) $-35.3^{\circ}$

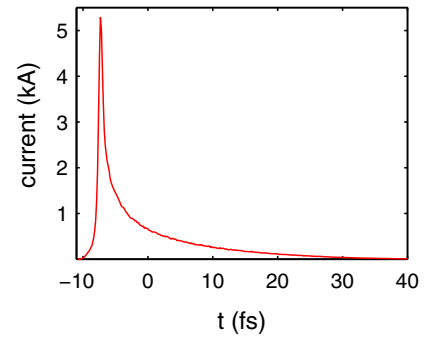

(d) $-35.4^{\circ}$

FIG. 4. ELEGANT tracking results of electron bunch distribution in the longitudinal phase space (upper) and its current profile (lower) at the LCLS undulator entrance for different L2 $\mathrm{rf}$ phases. The L1S rf phase is $-27^{\circ}$. L1X is with the phase of $-180^{\circ}$ and amplitude of $15 \mathrm{MV}$. 
decreased, a fast drop of the peak current can be observed [see Fig. 4(d)], since the full-compression region is moved from the core part of the beam to the high-energy tail part, where less particles contribute to the horn.

In ELEGANT simulations, a simple one-dimensional model is used for LSC [42]. Considering that LSC plays an important role in this work, the validity of the LSC model should be benchmarked. IMPACT-Z [43,44], which calculates the real three-dimensional space charge field, has been used as a cross-check for two different cases. The first is for a L2 $\mathrm{rf}$ phase of $-35.0^{\circ}$, around which the electron beam current is more stable against L2 $\mathrm{rf}$ phase jittering. The second is for a L2 $\mathrm{rf}$ phase of $-35.3^{\circ}$, in which case the beam longitudinal phase space rotates to make full compression near the high-energy tail region. One-to-one simulations have been performed where the number of macroparticles used is the same as the number of electrons in the beam $\left(1.25 \times 10^{8}\right)$. The results for both cases are shown in Fig. 5, which compare with Figs. 4(b) and 4(c) from ELEGANT. One can see from the figure that they agree with ELEGANT results very well in both the current profile and phase space distribution, which indicates that the simple LSC model used in ELEGANT is appropriate in our case.

We have also observed some density modulation from IMPACT-Z tracking results (the fine structures in Fig. 5). This is related to microbunching instability [42,45,46], where the electrons with some small amount of density modulation get energy-modulated, mostly due to collective effects such as LSC, CSR, and linac wakefields. The induced energy modulation is then converted to additional density modulation through longitudinal phase space
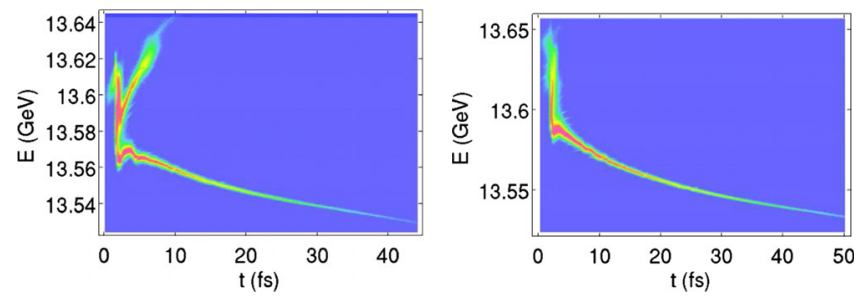

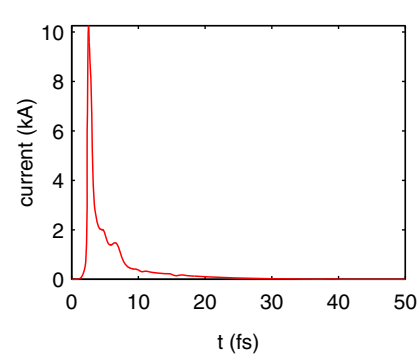

(a) $-35.0^{\circ}$

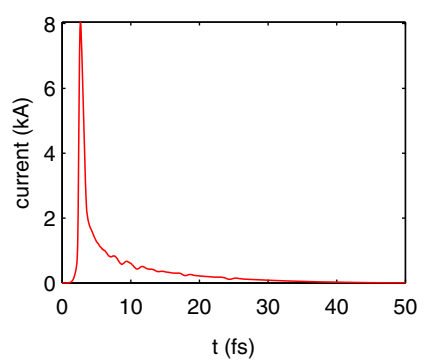

(b) $-35.3^{\circ}$
FIG. 5. IMPACT-Z tracking results of electron bunch distribution in the longitudinal phase space (upper) and its current profile (lower) at the LCLS undulator entrance in the case with the L2 $\mathrm{rf}$ phase of $-35.0^{\circ}$ (a) and $-35.3^{\circ}$ (b). The L1S rf phase is $-27^{\circ}$. L1X is with the rf phase of $-180^{\circ}$ and amplitude of $15 \mathrm{MV}$. rotation in bending magnets. In this work, the microbunching has very limited impact on the beam, especially around the horn, and its effect can therefore be ignored.

\section{FEL SIMULATION}

With such a short current spike generated from the optimized nonlinear compression scheme, we expect to produce ultrashort $\mathrm{x}$-ray pulses. Actually, this current spike is close to those generated in the enhanced selfamplified spontaneous emission scheme [6], but it is achieved here in a much simpler way. Using the ELEGANT dumped particles, we performed FEL simulations with the three-dimensional GENESIS 1.3 code [47]. The radiation wavelength for these particular simulations is chosen to be $1.5 \AA$, corresponding to an undulator strength parameter $K$ of 3.5 .

For this nonlinear compression, single-horn mode, the peak current of the electron beam is not sensitive to the L2 rf phase within the measured jitter range. However, the L2 rf phase still has some impact on the details of the current profile. Therefore, FEL simulations have been performed over a large range of L2 $\mathrm{rf}$ phases, from $-35.4^{\circ}$ to $-34.8^{\circ}$. A one-dimensional LSC model, similar to what is implemented in ELEGANT, has been included in the GENESIS simulations taking into account the electron beam transverse wiggling motion in an undulator [48]. Without any undulator taper, the simulated results of the FEL power profile and spectrum just at saturation $(60 \mathrm{~m})$ and deep saturation $(120 \mathrm{~m})$ are shown in Fig. 6, corresponding to the cases with a L2 $\mathrm{rf}$ phase of $-35.0^{\circ}$ [Fig. 4(b)] and $-35.3^{\circ}$ [Fig. 4(c)], respectively.

In Figs. 6(a) and 6(b), for the case with a L2 rf phase of $-35.0^{\circ}$, one can see that the FEL signal has multiple spikes in both the temporal and spectral profiles. The situation is much better for the case with a L2 $\mathrm{rf}$ phase of $-35.3^{\circ}$ [see Figs. 6(c) and 6(d)], where we can see the FEL power profile has one dominant spike. However, satellite peaks still emerge as the FEL interaction continues past saturation, and multiple spikes are clearly seen in the spectral plots.

To obtain a clean single-spike x-ray pulse, the chirp-taper idea could be applied in this scheme where the chirp is induced from LSC mainly after $\mathrm{BC} 2$ and gets stronger when the electrons wiggle inside the undulator. This timeenergy chirp, mainly within the horn region, grows in proportion to the distance along the undulator. As the radiation wave slips to the front of the horn, the energy of the electrons in the front part of the horn increases due to the LSC and offsets the resonant condition. By tapering the undulator strength parameter $K$, the energy change can be compensated to a large extent, and the resonant condition for the interacting part of the electrons can be preserved.

Since the strength of the LSC fields depends on the derivative of the electron current, other parts of the electrons with lower current would lead to a smaller chirp. 

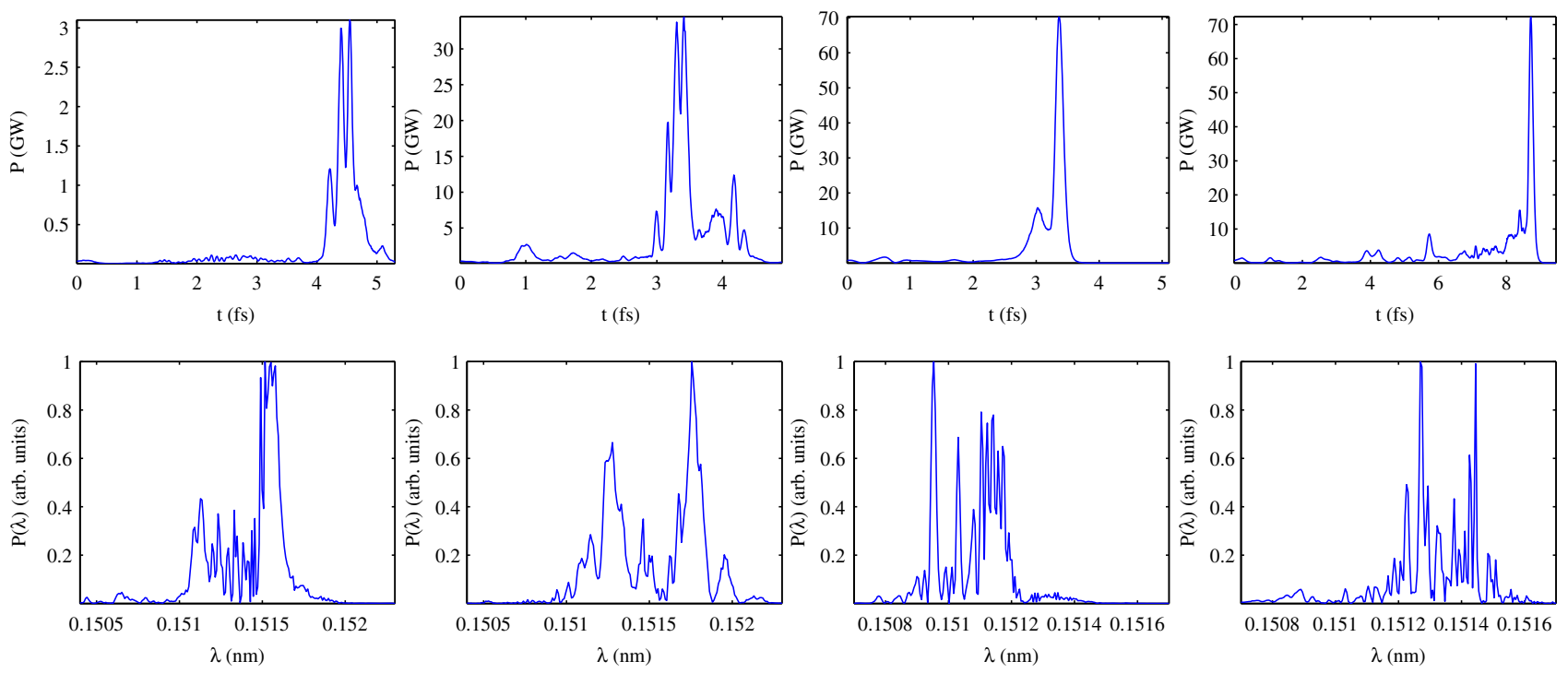

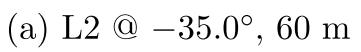

(b) L2@ $@ 35.0^{\circ}, 120 \mathrm{~m}$

(c) L2@ $-35.3^{\circ}, 60 \mathrm{~m}$

(d) L2@ $-35.3^{\circ}, 120 \mathrm{~m}$

FIG. 6. Simulation results of the FEL power profile (upper) and spectrum (lower) at different locations in the undulator using the electron beam with the $\mathrm{L} 2 \mathrm{rf}$ phase at $-35.0^{\circ}$ for (a) and (b) and the L2 $\mathrm{rf}$ phase at $-35.3^{\circ}$ for (c) and (d). No undulator taper is applied. In the time-domain $\mathrm{x}$-ray power profiles, the pulse head is to the right in these plots and throughout.

If the taper is chosen to match the strongest chirp around the peak of the horn, we can preserve the resonant condition in this region while simultaneously suppressing the FEL process elsewhere in the bunch. In addition, the larger current at the horn produces higher FEL gain than other parts. We applied the reverse taper in the following analyses and simulations.

In the limit when the electron bunch length in the average comoving frame is much larger than the transverse beam size, the LSC field can be estimated by using a simplified expression [49]:

$$
E_{z} \approx-\frac{Z_{0} I^{\prime}(s)}{4 \pi \bar{\gamma}_{z}^{2}}\left(2 \ln \frac{\bar{\gamma}_{z} \sigma_{z}}{r_{b}}\right)
$$

where $Z_{0}=377 \Omega, I^{\prime}(s)=d I / d s$ is the derivative of the electron current profile with respect to the longitudinal bunch coordinate $s, \bar{\gamma}_{z}=\gamma / \sqrt{1+K^{2} / 2}$ accounts for the reduction of the average longitudinal Lorentz factor due to the electron wiggling motion [48], $\gamma$ is the relativistic factor for the electron beam, $r_{b}$ is the beam radius of a uniform transverse distribution, and $\sigma_{z}$ is the rms bunch length.

We find that, when the L2 $\mathrm{rf}$ phase is around $-35.0^{\circ}$, the current profile of the electron bunch has a wide pent roof, leading to a discontinuous LSC field profile which is split into two fractions [see Fig. 7(a)]. As the L2 rf phase approaches $-35.3^{\circ}$, the current profile becomes smooth around the horn, and therefore the LSC field profile becomes simplified [see Fig. 7(b)]. During FEL operation, we have to set up a single taper that works with a jittering L2 rf phase. Considering that, in this study, the undulator taper is set according to the LSC field profile at $-35.0^{\circ}$, which is about the central phase of the expected operating point for this nonlinear compression mode.

To evaluate the taper, we used the formula in Refs. [7,30]:

$$
\frac{d \ln K}{d z}=-\frac{\lambda_{s}}{\lambda_{u}} \frac{2+K^{2}}{K^{2}} \cdot \frac{1}{c} \frac{d \ln \gamma}{d t},
$$

which relates the undulator taper $d \ln K / d z$ to the timeenergy chirp in the electron bunch, $d \ln \gamma / d t$, where $\lambda_{s}$ is the radiation wavelength, $\lambda_{u}$ is the undulator period, and $c$ is the speed of light. The equation can be rewritten in an approximate form:

$$
\frac{\Delta K / K}{\Delta z} \approx-\frac{\lambda_{s}}{\lambda_{u}}\left(1+\frac{2}{K^{2}}\right) \frac{\Delta E / E}{\Delta s},
$$

where the time-energy chirp within the bunch is represented as $\Delta E / E / \Delta s$ and $\Delta E / E$ is the relative energy change of electrons over a distance of $\Delta s$ along the bunch. When the LSC effect inside the undulator dominates the time-energy chirp, as in the cases discussed in this paper, $\Delta E / E / \Delta s$ can be evaluated from the LSC field using Eq. (1). As shown in Fig. 7, the LSC-induced time-energy chirp is not constant along the bunch, and we choose the undulator taper to match the maximum slope of the induced chirp. In this way, we expect to amplify the FEL mainly near the horn region. Since the chirp grows linearly along the distance, for simplification, we just calculate the chirp at half of the expected saturation length (about $30 \mathrm{~m}$ in this study) and apply a linear taper. With these assumptions, we 


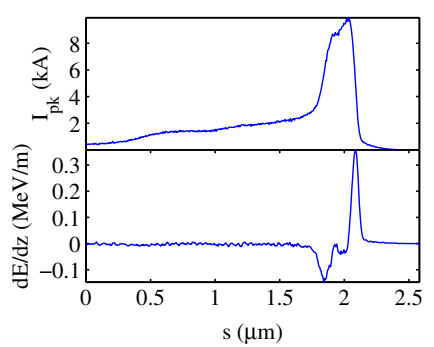

(a) $-35.0^{\circ}$

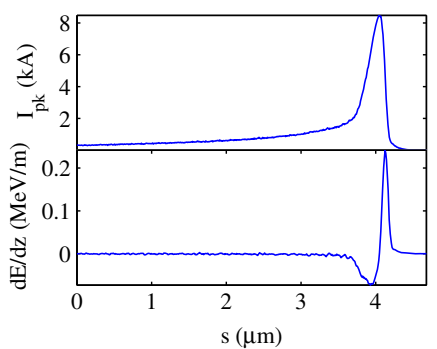

(b) $-35.3^{\circ}$
FIG. 7. Electron current profile (upper) and the energy modulation of electron bunch per unit length due to the LSC effect in the undulator (lower) in the cases with the L2 rf phase of $-35.0^{\circ}$ (a) and $-35.3^{\circ}$ (b). The electron bunch head is on the right-hand side.

get $\quad \Delta E / E / \Delta s=1.51 \times 10^{4} \mathrm{~m}^{-1} \quad$ and $\quad \Delta K / K / \Delta z=$ $-8.8 \times 10^{-5}$. If we taper the undulator from 20 to $132 \mathrm{~m}$, i.e., $\Delta z=112 \mathrm{~m}$, we then have $\Delta K / K \approx-1 \%$. One should note that $\Delta K$ in this paper is defined according to a convention in the FEL community. A negative $\Delta K$ means the value of $K$ increases along the undulator and is referred to as the reverse taper.

Figures 8 and 9 show the typical FEL power profile using a reverse taper of $-1 \%$ over the range from 20 to $132 \mathrm{~m}$ in the undulator. In Fig. 8, where the L2 rf phase is $-35.0^{\circ}$, we really obtained a single spike at $60 \mathrm{~m}$ in the undulator. As the FEL interaction continues, electrons on the tail part radiate, forming a small bump after the spike. The spectrum is relatively clean before $80 \mathrm{~m}$. However, after $80 \mathrm{~m}$, more contents show up. Nevertheless, the contrast ratio between the spike and the bump is more than a factor of 5 at the distance of $120 \mathrm{~m}$ in the undulator. Therefore, a single FEL spike still dominates, and the reverse taper really helps achieve a cleaner single spike.

In the case with the L2 $\mathrm{rf}$ phase of $-35.3^{\circ}$ (see Fig. 9), the single-spike feature is more noticeable. Lasing happens only in the horn region, while electrons in the other parts of the bunch are successfully prevented from lasing. One can see from the figure that even in the postsaturation regime, from about $60 \mathrm{~m}$ to the end, no other side spikes show up. The spectrum is clean until $\sim 100 \mathrm{~m}$. One should note that in other short-pulse schemes a single spike could typically be obtained right at saturation, while after saturation many side spikes will emerge. Here the chirp-taper scheme shows a great advantage of generating a short pulse while simultaneously suppressing the unwanted lasing even after FEL saturation. Therefore, the reverse undulator taper also helps tolerate the beam jittering for achieving a stable single-spike operational mode. However, compared to the case without tapering, the absolute peak power is reduced by a factor of 2 . It is worth emphasizing that the taper is evaluated for the case with the L 2 rf phase of $-35.0^{\circ}$, which is not the best choice for the case with the L $2 \mathrm{rf}$ phase of $-35.3^{\circ}$. However, our simulations indicate that the reverse taper of $-1 \%$ is the optimized choice for the $\mathrm{L} 2 \mathrm{rf}$ phase range from $-35.4^{\circ}$ to $-34.8^{\circ}$, which assures single-spike FEL radiation in most cases. We choose this $-1 \%$ linear taper for further statistical studies.

To analyze the statistical fluctuation on the radiation pulse, a series of 20 separate GENESIS runs have been done, distinguished by different random number seeds for shot noise initialization. Figure 10 shows the FEL power and pulse duration (FWHM) along the undulator when the
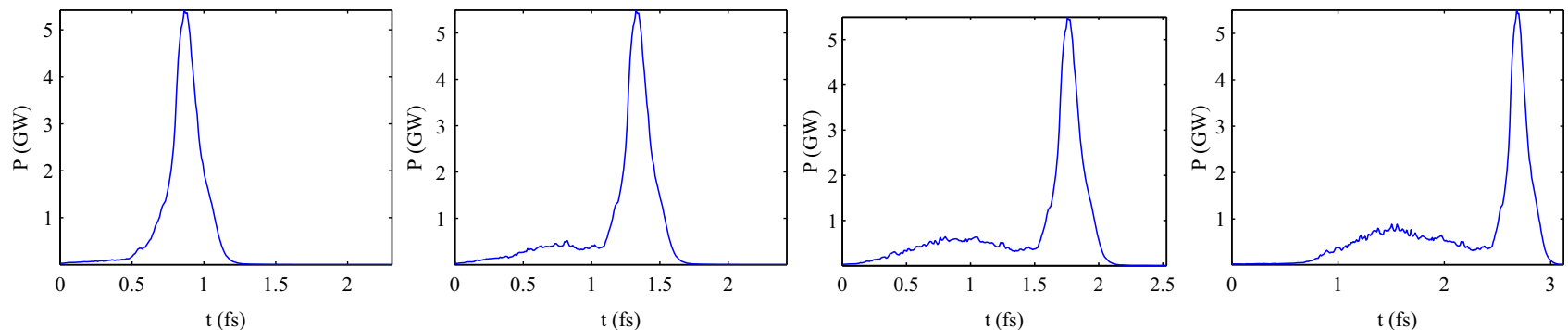

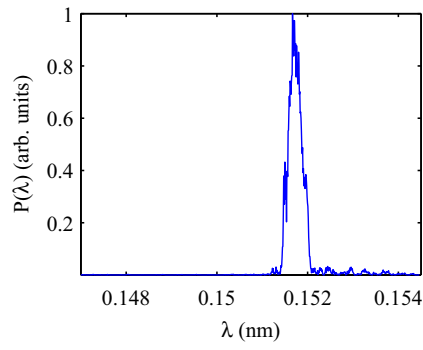

(a) $60 \mathrm{~m}$

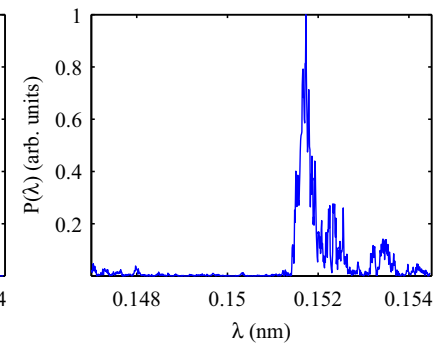

(b) $80 \mathrm{~m}$

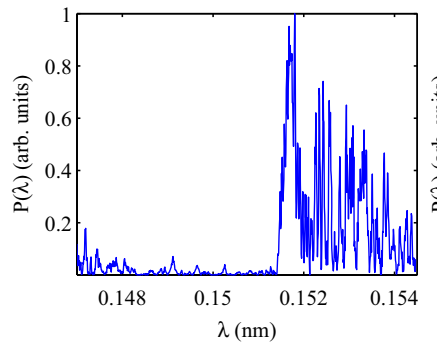

(c) $100 \mathrm{~m}$

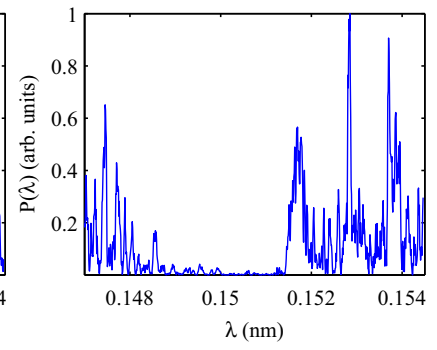

(d) $120 \mathrm{~m}$

FIG. 8. Simulation results of the FEL pulse (upper) and spectrum (lower) at different locations in the undulator using the electron beam in Fig. 4(b), where the L2 rf phase is $-35.0^{\circ}$ and the undulator is tapered at $\Delta K / K=-1 \%$ from 20 to $132 \mathrm{~m}$. 

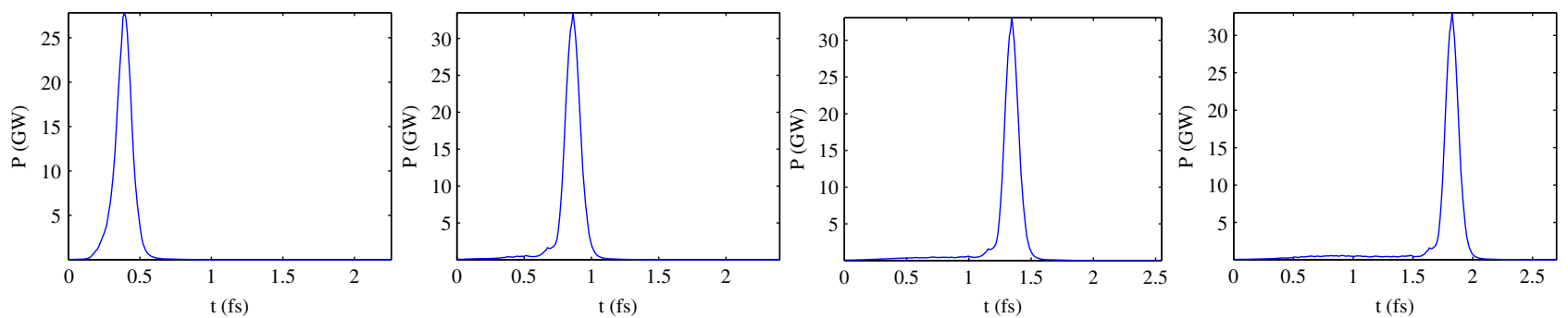

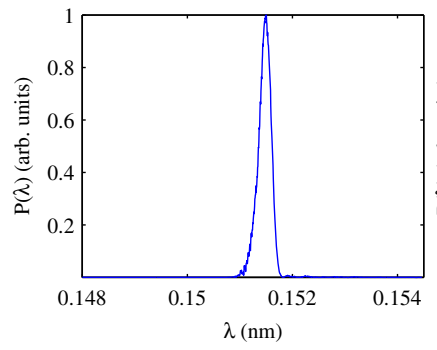

(a) $60 \mathrm{~m}$

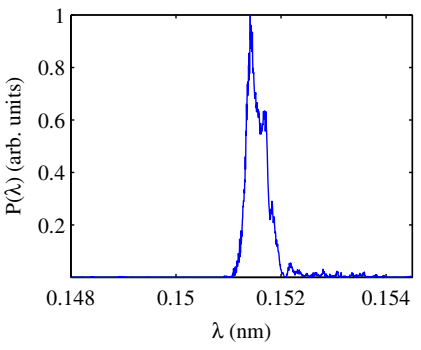

(b) $80 \mathrm{~m}$

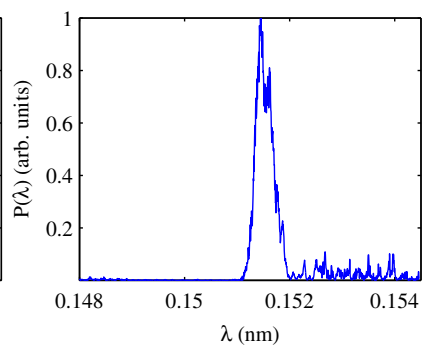

(c) $100 \mathrm{~m}$

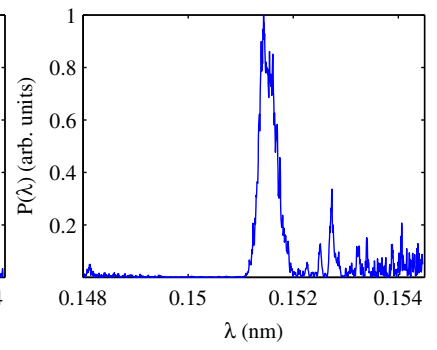

(d) $120 \mathrm{~m}$

FIG. 9. Simulation results on the FEL power profile (upper) and spectrum (lower) at different locations in the undulator using the electron beam in Fig. 4(b), where the L2 $\mathrm{rf}$ phase is $-35.3^{\circ}$ and the undulator is tapered at $\Delta K / K=-1 \%$ from 20 to $132 \mathrm{~m}$.

L2 rf phase is at $-35.3^{\circ}$, which is averaged over the 20 shots. We can see from the figure that the FEL saturates around the distance of $60 \mathrm{~m}$ in the undulator with the power of about $35 \mathrm{GW}$, and the pulse duration is below 140 as.

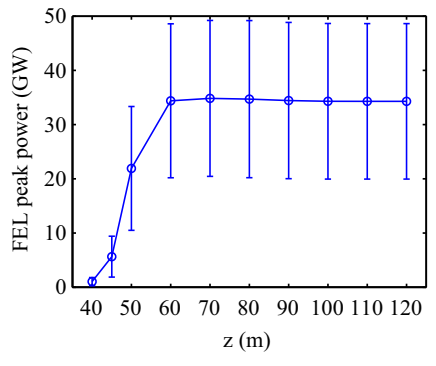

(a)

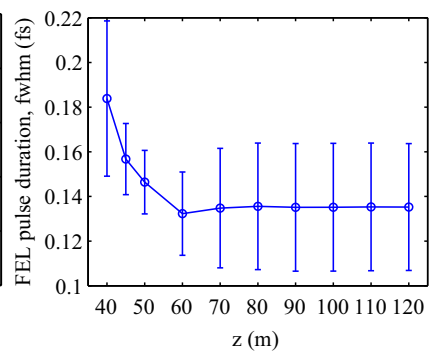

(b)
FIG. 10. FEL peak power (a) and pulse duration (b) along the undulator. The $\mathrm{L} 2 \mathrm{rf}$ phase is $-35.3^{\circ}$, and the undulator is tapered at $\Delta K / K=-1 \%$ from 20 to $132 \mathrm{~m}$.

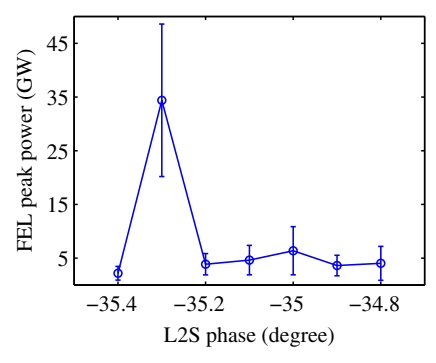

(a)

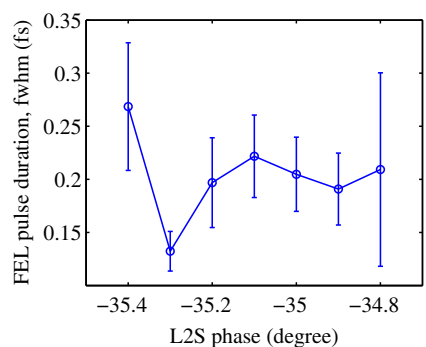

(b)
FIG. 11. FEL peak power (a) and pulse width (b) at $60 \mathrm{~m}$ in the undulator vs the L2 $\mathrm{rf}$ phase. The undulator is tapered at $\Delta K / K=-1 \%$ from 20 to $132 \mathrm{~m}$.
Since the self-amplified spontaneous emission FEL starts from noise, both the peak power and pulse duration fluctuate, with an rms value of $15 \mathrm{GW}$ and 20 as, respectively, at the distance of $60 \mathrm{~m}$ in the undulator.

Figure 11 shows the averaged FEL peak power and pulse duration (FWHM) as a function of the L2 rf phase, using the same fixed $-1 \%$ taper. The figure indicates that, when the L2 $\mathrm{rf}$ phase is at $-35.3^{\circ}$, the FEL has the maximum peak power and the minimum pulse duration. However, this point is not stable, since the FEL power has a large standard deviation and drops quickly to a few gigawatts as the $\mathrm{L} 2 \mathrm{rf}$ phase changes (jitters) by $\pm 0.1^{\circ}$. One can also see from the figure that, when the L2 $\mathrm{rf}$ phase is around $-35.0^{\circ}$, the FEL power is more stable against L2 $\mathrm{rf}$ phase jittering. This suggests that we may have two different regimes for FEL operation. One is around $-35.3^{\circ}$ (within the range from $-35.4^{\circ}$ to $-35.2^{\circ}$ ), in which case the FEL power is higher; however, the power fluctuation will be larger and sensitive to L2 $\mathrm{rf}$ phase jittering. The other is around $-35.0^{\circ}$ (within the range from $-35.2^{\circ}$ to $-34.8^{\circ}$ ), in which case the FEL power will be lower; however, it is much more stable within the measured L2 $\mathrm{rf}$ phase jitter range of $0.1 \mathrm{fs}$ rms [39]. In this case, the FEL pulse duration is around 200 as. It should be pointed out that at some data point, e.g., the L $2 \mathrm{rf}$ phase of $-34.8^{\circ}$, a few shots have two distinct spikes. Therefore, the corresponding data points in the figure have a larger error bar in FEL pulse duration.

\section{SUMMARY AND DISCUSSION}

Based upon the current hardware of the LCLS, generating hard X-ray FEL radiation with subfemtosecond pulse duration has been studied. This has been achieved in simulation by using two procedures. First, the nonlinear 
compression of a low-charge $(20 \mathrm{pC})$ electron beam is optimized to get a single-horn current profile with a FWHM of about $1 \mathrm{fs}$. Second, the LSC-induced time-energy chirp of the electron beam is leveraged by reversely tapering the undulator field strength allowing only a fraction of electrons around the horn of the bunch to lase, thus leading to very short $\mathrm{x}$-ray pulses with a single spike.

The simulations suggest that, for the nonlinear compression single-spike mode reported in this paper, we may have two different FEL operation regimes, distinguished by the L2 rf phase. For the first regime, with the L2 rf phase around $-35.3^{\circ}$, the FEL power of about $35 \mathrm{GW}$ and pulse duration below 140 as FWHM can be obtained. However, the FEL power fluctuates about $\pm 15 \mathrm{GW}$, and it is sensitive to L2 rf phase jittering (within $\pm 0.1^{\circ}$ ). For the second regime, with the L2 $\mathrm{rf}$ phase around $-35.0^{\circ}$, the FEL power is around $4 \mathrm{GW}$ and the pulse duration is about 200 as FWHM. In this latter regime, the FEL is not sensitive to L2 rf phase variation, and a jitter of $\pm 0.2^{\circ}$ can be tolerated. This is well within the measured LCLS linac rf phase jittering range, which provides a stable single-spike operation mode. Since the detailed phase space distribution would affect the FEL performance, some further optimizing of the L1X rf phase or amplitude could be done in the future, and it is a compromise between the FEL peak power and stability.

Experimental demonstration of this scheme has been planned at the LCLS, and the diagnostics on subfemtosecond time scales is very challenging. The high-resolution spectrometer [50] would be the first step to verify these single-spike pulses in the frequency domain. Also note that this same scheme can be applied to soft x-ray FELs with an expected pulse duration about a few femtoseconds.

\section{ACKNOWLEDGMENTS}

The authors thank G. Marcus for helpful discussions on this topic and for carefully reading the manuscript. This work is supported by the U.S. Department of Energy Contracts No. DE-AC02-76SF00515 and No. DE-AC0205CH11231 and Major State Basic Research Development Program of China (Grant No. 2011CB808301).

[1] P. Emma et al., Nat. Photonics 4, 641 (2010).

[2] T. Ishikawa et al., Nat. Photonics 6, 540 (2012).

[3] A. A. Zholents and W. M. Fawley, Phys. Rev. Lett. 92, 224801 (2004).

[4] E. L. Saldin, E. A. Schneidmiller, and M. V. Yurkov, Opt. Commun. 237, 153 (2004).

[5] E. L. Saldin, E. A. Schneidmiller, and M. V. Yurkov, Opt. Commun. 239, 161 (2004).

[6] A. A. Zholents and G. Penn, Phys. Rev. ST Accel. Beams 8, 050704 (2005).

[7] E. L. Saldin, E. A. Schneidmiller, and M. V. Yurkov, Phys. Rev. ST Accel. Beams 9, 050702 (2006).
[8] A. A. Zholents and M. S. Zolotorev, New J. Phys. 10, 025005 (2008).

[9] Y. Ding, Z. Huang, D. Ratner, P. Bucksbaum, and H. Merdji, Phys. Rev. ST Accel. Beams 12, 060703 (2009).

[10] T. Tanaka, Phys. Rev. Lett. 110, 084801 (2013).

[11] J. Qiang and J. Wu, Appl. Phys. Lett. 99, 081101 (2011).

[12] J. Qiang and J. Wu, J. Mod. Opt. 58, 1452 (2011).

[13] P. Emma, Z. Huang, and M. Borland, in Proceedings of the 26th International Free Electron Laser Conference and 11th FEL Users Workshop, FEL2004, Trieste, Italy, 2004 (Comitato Conferenze Elettra, Trieste, Italy, 2004), p. 333.

[14] J. B. Rosenzweig et al., Nucl. Instrum. Methods Phys. Res., Sect. A 593, 39 (2008).

[15] S. Reiche, P. Musumeci, C. Pellegrini, and J. B. Rosenzweig, Nucl. Instrum. Methods Phys. Res., Sect. A 593, 45 (2008).

[16] V. Wacker et al., in Proceedings of the 34th International Free Electron Laser Conference, FEL2012, Nara, Japan, 2012 (JACoW, Nara, Japan, 2012), p. 606.

[17] Y. Ding et al., Phys. Rev. Lett. 102, 254801 (2009).

[18] C. Behrens et al., Nat. Commun. 5, 3762 (2014).

[19] Y. Ding et al., Phys. Rev. Lett. 109, 254802 (2012).

[20] Y. Ding et al., in Proceedings of the 4th International Particle Accelerator Conference, IPAC2013, Shanghai, China, 2013 (JACoW, Shanghai, China, 2013), p. 1316.

[21] B. Beutner and S. Reiche, in Proceedings of the 33rd International Free Electron Laser Conference, FEL2011, Shanghai, China, 2011 (EPS-AG, Shanghai, China, 2011), p. 235.

[22] B. Marchetti, M. Krasilnikov, F. Stephan, and I. Zagorodnov, Phys. Procedia 52, 80 (2014).

[23] E. L. Saldin, E. A. Schneidmiller, and M. V. Yurkov, Opt. Commun. 212, 377 (2002).

[24] D. Xiang, Z. Huang, and G. Stupakov, Phys. Rev. ST Accel. Beams 12, 060701 (2009).

[25] A. Zholents and G. Penn, Nucl. Instrum. Methods Phys. Res., Sect. A 612, 254 (2010).

[26] J. Yan, H. Deng, D. Wang, and Z. Dai, Nucl. Instrum. Methods Phys. Res., Sect. A 621, 97 (2010).

[27] N. R. Thompson and B. W. J. McNeil, Phys. Rev. Lett. 100, 203901 (2008).

[28] D. J. Dunning, B. W. J. McNeil, and N. R. Thompson, Phys. Rev. Lett. 110, 104801 (2013).

[29] C. Feng, J. Chen, and Z. Zhao, Phys. Rev. ST Accel. Beams 15, 080703 (2012).

[30] W. M. Fawley, Nucl. Instrum. Methods Phys. Res., Sect. A 593, 111 (2008).

[31] L. Giannessi et al., Phys. Rev. Lett. 106, 144801 (2011).

[32] G. Marcus et al., Appl. Phys. Lett. 101, 134102 (2012).

[33] P. Emma, LCLS technical note, Report No. SLAC-TN-05004, 2001.

[34] K. Floettmann, T. Limberg, and Ph. Piot, Report No. TESLA-FEL-01-06, DESY-HH, 2001.

[35] M. Dohlus, K. Flöttmann, O. S. Kozlov, T. Limberg, Ph. Piot, E. L. Saldin, E. A. Schneidmiller, and M. V. Yurkov, Nucl. Instrum. Methods Phys. Res., Sect. A 530, 217 (2004).

[36] L. Wang, Y. Ding, and Z. Huang, in Proceedings of the 2nd International Particle Accelerator Conference, IPAC2011, San Sebastián, Spain, 2011 (EPS-AG, Spain, 2011), p. 3131. 
[37] R. Akre et al., Phys. Rev. ST Accel. Beams 11, 030703 (2008).

[38] K. Bane and P. Emma, in Proceedings of the 21st Particle Accelerator Conference, PAC2005, Knoxville, TN, U.S., 2005 (IEEE, Piscataway, NJ, 2005), p. 4266.

[39] F.-J. Decker et al., in Proceedings of the 1st International Particle Accelerator Conference, IPAC2010, Kyoto, Japan, 2010 (ICR, Kyoto, 2010), p. 2296.

[40] J. Qiang, S. Lidia, R. Ryne, and C. Limborg-Deprey, Phys. Rev. ST Accel. Beams 9, 044204 (2006).

[41] M. Borland, Advanced Photon Source Report No. LS-287, 2000.

[42] Z. Huang, M. Borland, P. Emma, J. Wu, C. Limborg, G. Stupakov, and J. Welch, Phys. Rev. ST Accel. Beams 7, 074401 (2004).

[43] J. Qiang, R. D. Ryne, S. Habib, and V Decyk, J. Comput. Phys. 163, 434 (2000).
[44] J. Qiang, R. D. Ryne, M. Venturini, and A. A. Zholents, Phys. Rev. ST Accel. Beams 12, 100702 (2009).

[45] M. Borland et al., Nucl. Instrum. Methods Phys. Res., Sect. A 483, 268 (2002).

[46] E. L. Saldin, E. A. Schneidmiller, and M. V. Yurkov, Nucl. Instrum. Methods Phys. Res., Sect. A 528, 355 (2004).

[47] S. Reiche, Nucl. Instrum. Methods Phys. Res., Sect. A 429, 243 (1999).

[48] G. Geloni, Nucl. Instrum. Methods Phys. Res., Sect. A 583, 228 (2007).

[49] A.W. Chao, Physics of Collective Beam Instabilities in High Energy Accelerators (Wiley, New York, 1993).

[50] D. Zhu, M. Cammarata, J. M. Feldkamp, D. M. Fritz, J. B. Hastings, S. Lee, H. T. Lemke, A. Robert, J. L. Turner, and Y. Feng, Appl. Phys. Lett. 101, 034103 (2012). 